\title{
Indoleamine 2,3-dioxygenase and regulatory dendritic cells contribute to the allograft protection induced by infusion of donor-specific splenic stromal cells
}

\author{
Li Liu, Lihua Duan, Min Gong, Hong Dai, Quan Gong, Fang Zheng, Zheng Tan, Congyi Wang, Feili Gong \\ and Min Fang
}

Cellular \& Molecular Immunology (2011) 8, 92; doi:10.1038/cmi.2010.61

Correction to: Cellular \& Molecular Immunology advance online publication 8 November 2010; doi:10.1038/cmi.2010.53

The html and pdf versions of this paper have been corrected. The printed version of the paper also carries the correct information.

In page 1 , the reference number ' 19 ' in the line: 'In the context of transplantation, we have previously shown that over expression of IDO induces a protective effect against rejection of allergenic grafts by inhibiting alloreactive T-cell activities. ${ }^{17,19}$, has been deleted.

In page 6 , the reference number ' 16,18 ' in the line: 'Because we have previously demonstrated that IDO plays a role in allograft tolerance, ${ }^{16,18}$ we tested whether IDO is essential for the protection of skin allografts by infusion of donor-derived SSCs.' has been changed to ' 17,19 '.

Figure $7 \mathrm{a}$ in the above paper appeared incorrectly. One asterisk in the graph in Figure 7a is missing. The correct graph, which is shown as below, has been used to replace the incorrect one.

The content of reference 24 has been changed to: Munn DH, Sharma MD, Hou D, Baban B, Lee JR, Antonia SJ et al. Expression of indoleamine 2,3-dioxygenase by plasmacytoid dendritic cells in tumor-draining lymph nodes. J Clin Invest 2004; 114: 280-290.

The content of reference 25 has been changed to: Morelli AE, Thomson AW. Tolerogenic dendritic cells and the quest for transplant tolerance. Nat Rev Immunol 2007; 7: 610-621.

The editorial office would like to apologize for the mistakes.

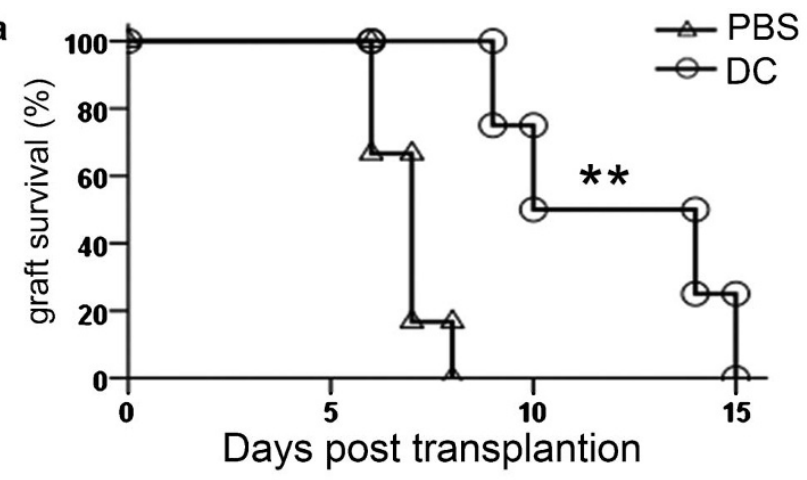

b
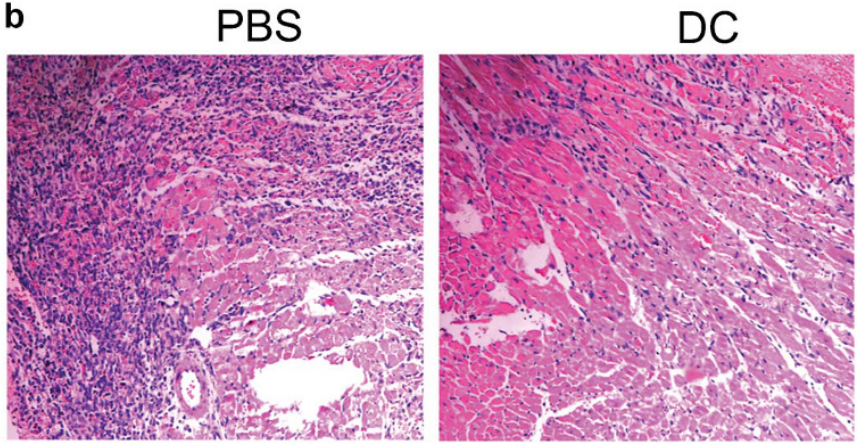

Figure 7 Intravenous infusion of SSC-derived DCs prolongs the survival of heart allografts and attenuates the infiltrate of leukocytes into the grafts. (a) C57BL/6 mouse recipients were treated with intravenous infusion of either $2 \times 10^{5}$ SSCderived DCs from BALB/c mice per mouse or PBS 7 days prior to transplantation. Subsequently, the mice were transplanted with BALB/c heart grafts ( $n=6-8$ mice per group). The MST of the grafts was monitored. ${ }^{* *} P<0.01$. (b) Histological analysis of heart allografts after transplantation revealed a reduced level of allograft leukocytes infiltration and necrosis in the SSC-derived DC-treated group compared to that in the PBS-treated group $(\times 200)$. The data shown are representative of three independent experiments. DC, dendritic cell; MST, mean survival time; PBS, phosphate-buffered saline; SSC, splenic stromal cell. 